\title{
A thermal-elastic analysis on laser-induced reverse bulging and plugging in circular brass foil
}

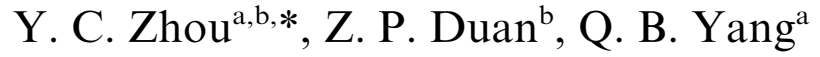 \\ a Department of Physics, Xiangtan University, 411105, Xiangtan Hunan, P.R. China $\dagger$ \\ ${ }^{\mathrm{b}}$ Laboratory for Laser and Dynamic Behaviors of Materials, Institute of Mechanics, CAS, Beijing 100080, P.R. China
}

Received 18 December 1996; in revised form 10 June 1997

\begin{abstract}
A new failure mode is observed in circular brass foils induced by laser beam. The new failure is based on the following experimental facts: (1) the peripheries of the circular brass foils are fixed and the surfaces of the foils are radiated by laser beam; (2) the laser beam used is considered to be non-Gaussian spatially, actually an approximately uniform distribution limited in a certain size spot; (3) the pulse on time of laser beam should be $250 \mu$ s, i.e. so called long duration pulse laser. The failure process consists of three stages; i.e. thermal bulging, localized shear deformation and perforation by plugging. The word 'reverse' in 'reverse bulging and plugging mode' means that bulging and plugging occur in the direction of incident laser beam. To study the newly-discovered type of failure quantitatively, analytical solutions for the axisymmetric temperature field and deflection curve are derived. The calculated results show that the newly discovered failure mode is attributed to the spatial structure effect of laser beam indeed. (C) 1998 Elsevier Science Ltd. All rights reserved.
\end{abstract}

\section{Introduction}

A great deal of attention has been paid to high power laser in the fields of materials and/or structure damage and lasers processing for the last several years. Various type modes of material damage such as spallation, melting and/or vaporization (Kar and Mazumder, 1990) could occur depending on laser parameters, for example laser power, laser beam diameter, pulse on time, etc. When the intensity of the continuous wave laser is about $10^{3} \mathrm{~W} / \mathrm{cm}^{2}$, there is the potential of failure by thermal stress (Garrison, 1976). The damage may occur by melting and/or vaporization for the laser intensity of the order of $10^{5}-10^{8} \mathrm{~W} / \mathrm{cm}^{2}$ and the pulse duration of the order of milliseconds (Chan and Mazumder, 1987; Smurov et al. 1992; Zweig, 1991). In this case, the thermal stress

\footnotetext{
* Corresponding author.

$\dagger$ Mailing address.
} 


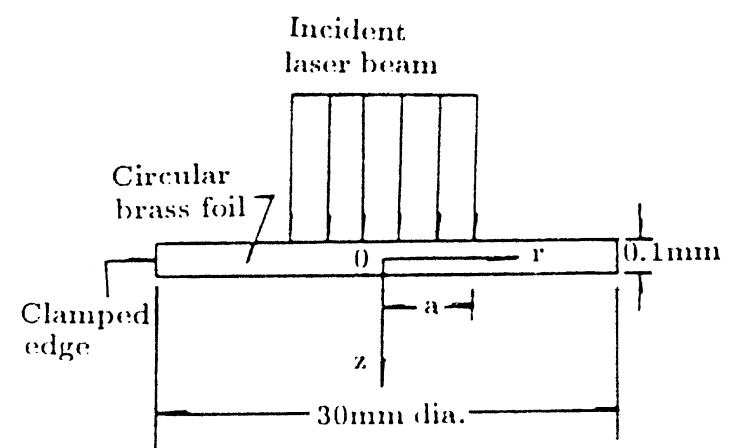

Fig. 1. Schematic diagram of a normal incident laser beam impinging on a circular brass foil specimen.

concentrated around the crater plays an important role in material and/or structure damage. The formation of plasma may generate shock waves propagating into the materials for the laser intensity of the order of $10^{8}-10^{10} \mathrm{~W} / \mathrm{cm}^{2}$ and the pulse duration of the order of nanoseconds. When wave reflection takes place between two surfaces, there is the potential of failure by spallation (Eliezer et al., 1990; Boustie and Cottet, 1991; Larson, 1975; Fox and Barr, 1973).

Besides the failure modes mentioned above, we will propose a new mode in the present paper which is characterized by non-Gaussian spatial distribution and long pulse on time of incident laser beam.

In this paper, the laser intensity threshold for the new failure mode is presented and the macroscopical phenomena are briefly described in Section 2. The detailed results of the microscopical observations have been presented (Zhou et al., 1997; Duan et al, 1994). The experimental results and analytical solution for the axisymmetric temperature distributions are found in Section 3. Although the shear strain concentration in the laser spot edge region causes the deformation to be plastic, the deformation is elastic at the early stage of laser irradiation. In this paper, only the reverse bulging is analyzed by using the temperature solutions and the classical Kirchhoff plate theory. Under the assumption of thermal-mechanical decoupling the deflection curve, i.e., the thermal bulging can be determined analytically in Section 4. Section 5 is dedicated to the conclusions. The detailed derivations of temperature distribution and dynamical deflection $w_{d}$ are given in Appendix A and B, respectively, for explicitness.

\section{Experimental procedure and observations}

\subsection{Experimental procedure}

Let an incident laser beam impinge normally on a circular brass foil as shown in Fig. 1, where $z$ - and $r$-direction are aligned normal and parallel to the specimen, respectively, whose edge is clamped, $2 a$ is the diameter of the laser spot with size $2-6 \mathrm{~mm}$. The foil has a diameter of $30 \mathrm{~mm}$ and thickness of $0.1 \mathrm{~mm}$ and is composed of $65 \%$ copper, $33.6 \%$ zinc, $0.03 \%$ iron, $0.06 \%$ antimony and other microelements that are negligibly small in percentage by weight. 


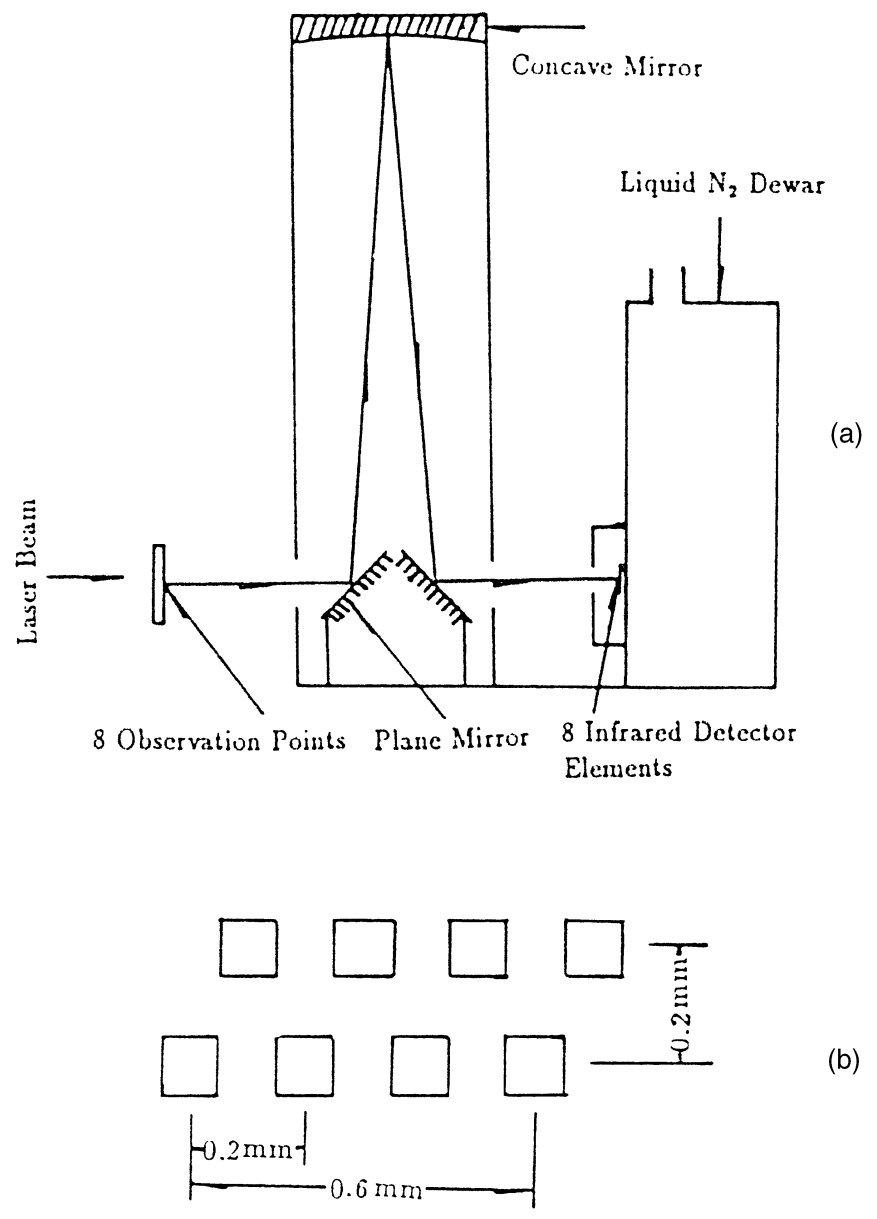

Fig. 2. Schematic diamgram of temperature measurements with high speed response infrared detectors: (a) optical path in the test; (b) devices arrangement.

The laser beam used is a single pulse $\mathrm{Nd}$ : glass with a wavelength of $1.06 \mu \mathrm{m}$, intensity order of $10^{5}-10^{6} \mathrm{~W} / \mathrm{cm}^{2}$ and energy from $25-40 \mathrm{~J}$. The diagnostics of the laser parameters provide a traditional monitoring of the laser beam characteristics, such as energy, temporal and spatial shapes. In the experiment, temperature rise on the rear surface of samples during laser irradiation is measured by a focused, imaging system of infrared (IR) detectors, of which the optic path system is designed to be different from that used by Duffy and coworkers (Hartley et al., 1987), Zehnder and Rosakis (Zehnder and Rosakis, 1991). The particular system consists of eight $0.1 \times 0.1 \mathrm{~mm}$ indium antimonide ( $\mathrm{InSb}$ ) infrared detectors mounted on a liquid nitrogen dewar as shown schematically in Fig. 2 . These detectors have high speed response ( $2 \mu$ s rise time) and are relatively easy to calibrate and use. Each detector is connected to an amplifier and the amplified signals are recorded on a high speed digital oscilloscope. The irradiation emitted from the rear surface of the specimen due to temperature rise is sensed by the detectors. The detector-mirror system was 


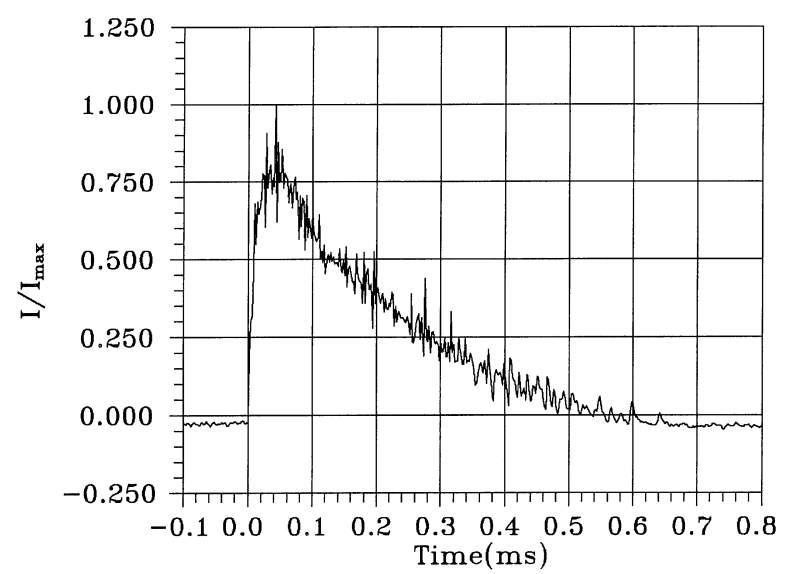

Fig. 3. Temporal shape of $\mathrm{Nd}$ : glass pulsed laser intensity.

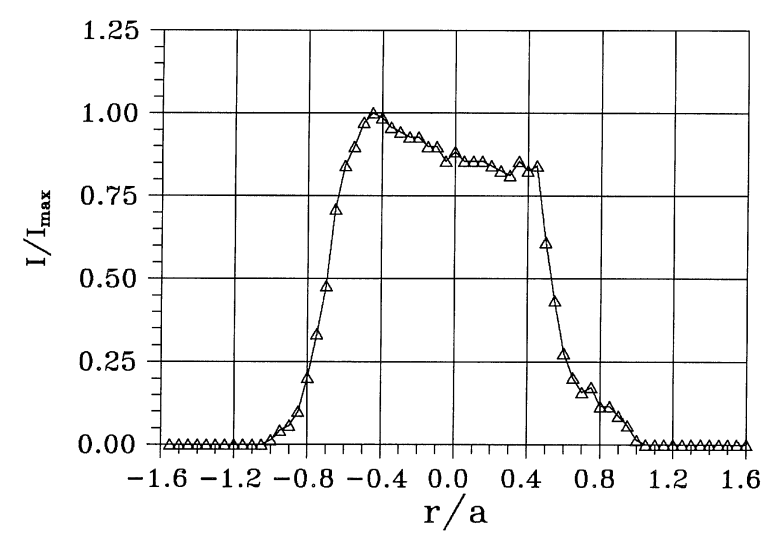

Fig. 4. Spatial shape of $\mathrm{Nd}$ : glass pulsed laser intensity.

calibrated before and after each series of tests. The first step in the calibration process is to measure the infrared radiation emitted from specimen heated internally with a soldering iron, while an imbedded thermocouple is used to determine its temperature. To produce an AC signal, a rotating notched disk was placed between the specimen and the detector in the calibration process.

The normalized laser intensity $I / I_{\max }$ vs time and space coordinates $r / a$ is shown in Figs 3 and 4, respectively, where $r$ and $a$ are the radial distance and the radius of the laser spot, respectively. The full time width at half maximum of the laser is approximately $250 \mu \mathrm{s}$. The laser intensity rises rapidly within $50 \mu$ s and then decays exponentially with a sawtooth oscillation. The spatial distribution of the laser intensity is non-Gaussian and roughly uniform within the laser irradiated region and declines very sharply towards the edge where the laser spot terminates. For the convenience of numerical analysis, the laser intensity $I$ is approximated by 


$$
I=I_{\max } e^{-\alpha t}\left(1-e^{-\beta t}\right) f(r)=I_{\max } g(t) f(r)
$$

where $\alpha$ and $\beta$ are determined experimentally test, and equal to $1.5 \times 10^{4} / \mathrm{s}$ and $8.0 \times 10^{4} / \mathrm{s}$, respectively. Therefore, laser energy $E_{J}=\beta \pi a^{2} I_{\max } / \alpha(\alpha+\beta)$ and we have

$$
f(r)= \begin{cases}1, & \text { if } 0 \leqslant r \leqslant a \\ 0, & \text { if } a<r<\infty\end{cases}
$$

and $f(r)=e^{-(r / a)^{2}}$ which are characterized by the non-Gaussian and Gaussian nature of the laser beam, respectively.

\subsection{Experimental results}

\subsubsection{Description of failure mode}

The evolution of specimen failure is illustrated schematically in Fig. 5. Figure 5(a) shows bulging of the brass foil at the earlier stage of laser irradiation. Note that this occurs towards the side of the incident laser beam where the temperature would be higher. Significant shear deformation occurs around a rim near the outer edge of the laser beam which is shown in Fig. 5(b). This leads to the softening of the material due to intense heating.

Further intensification of the energy around the periphery of the laser beam leads to the initiation of plugging and final perforation which are shown in Fig. 5(c)-(d), respectively. The plugging mode of failure is customarily known to be associated with metal projectiles penetrating through metal targets in plate form (e.g. Shoukry et al., 1991; Anderson and Bodner, 1988; Liss et al., 1983; Jenq et al., 1988). A plug of the target material is normally ejected in the direction of the energy source that is the moving projectile. While in the present case of laser induced the new failure mode, the plug is normally ejected in the direction opposite to the incident laser beam. The initial bulging occurs on the side with higher temperature that determines the direction of plugging.

\subsubsection{Threshold intensity}

According to the test data in this study, when the laser energy density is lower than $151 \mathrm{~J} / \mathrm{cm}^{2}$, or equivalently, the laser intensity is less than $0.51 \times 10^{6} \mathrm{~W} / \mathrm{cm}^{2}$, no visible macroscopic damage was observed on the front or rear surface of the brass specimen. When the laser intensity is increased to $0.56 \times 10^{6} \mathrm{~W} / \mathrm{cm}^{2}$, the bulging of the brass specimen is observed. A slight melting is observed on the front surface at the periphery of the laser spot. The laser intensity threshold value $I_{\text {cr }}$ for plugging to occur is about $0.61 \times 10^{6} \mathrm{~W} / \mathrm{cm}^{2}$. As $I$ exceeds $I_{\text {cr }}$, local melting of the material begins to take place. When the laser intensity is increased to $0.75 \times 10^{6} \mathrm{~W} / \mathrm{cm}^{2}$, the brass foil is totally fractured and fragmented. A study on laser-induced spallation in $0.1 \mathrm{~mm}$ thick copper foil has been made by Eliezer and coworkers (Eliezer et al., 1990). The intensity for spallation was of the order of $5.3 \times 10^{10} \mathrm{~W} / \mathrm{cm}^{2}$ which was five orders of magnitude larger than that for failure by plugging which was about $0.71 \times 10^{6} \mathrm{~W} / \mathrm{cm}^{2}$. The thresholds of the laser energy for the two different failure modes are almost not distinguishable and they are both approximately equal to $210 \mathrm{~J} / \mathrm{cm}^{2}$.

\subsubsection{Damage evolution}

Scanning electron microscopy (SEM) showed that increased laser intensity led to the initiation of microcracks in the peripheral region with radius $r \sim a$. The macrocracks appeared on the rear 


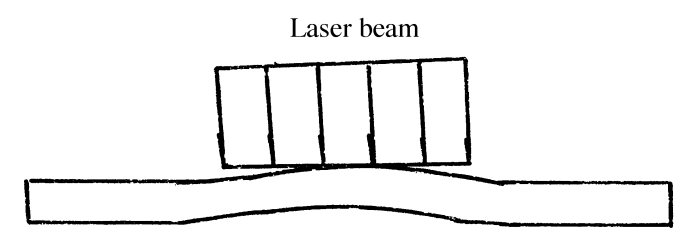

(a) Bulging

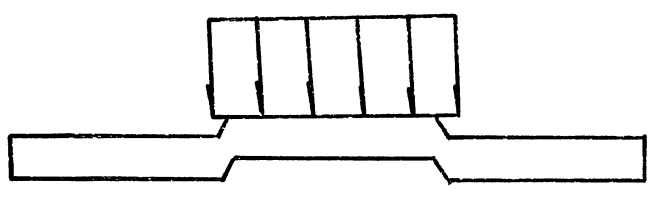

(b) Localized shear deformation

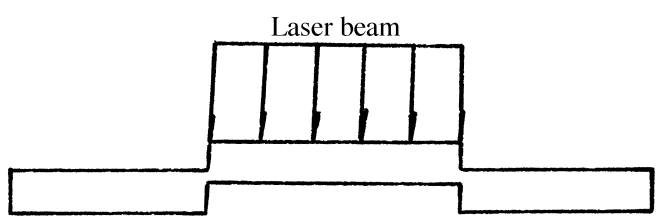

(c)Plugging initiation

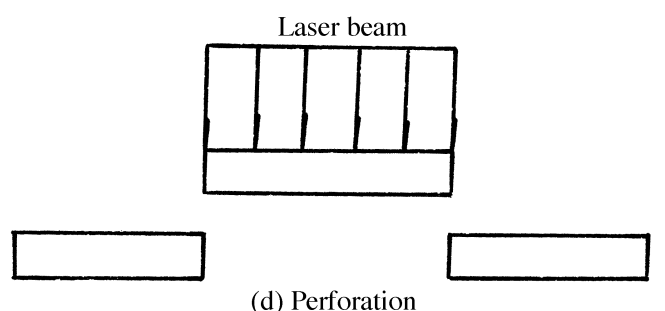

(d) Perforation

Fig. 5. Schematic illustration of damage evolution: (a) bulging; (b) localized shear deformation; (c) plugging initiation ; and (d) perforation.

surface and then spread into the material. They would grow and coalesce into macrocracks that grow rapidly in the circumferential direction as well as in the thickness direction. The macroscopical damage evolutions are shown in Figs 6,7 and 8 which, respectively, correspond to the schematic illustration of damage evolutions as shown in Fig. 5(a), (c) and (d). The photograph of a polished section of brass specimen is shown in Fig. 6. Bulging in the direction opposite to the incident laser beam is observed with a maximum center deflection of $0.03 \mathrm{~mm}$ which corresponds to a laser energy of $8.2 \mathrm{~J}$; an intensity greater than $0.61 \times 10^{6} \mathrm{~W} / \mathrm{cm}^{2}$; and a spot diameter of $2.3 \mathrm{~mm}$. Softening of the material begins around the outer edge of the laser spot.

When the laser intensity is increased to $0.61 \times 10^{6} \mathrm{~W} / \mathrm{cm}^{2}$, the plugging begins to take place in the brass foil. Figure 7 shows the circular brass foil failed by plugging opposite to the direction of the incident laser beam. This photograph of brass foil failure by plugging is viewed from the rear surface with a laser energy of $29 \mathrm{~J}$ over region $4.5 \mathrm{~mm}$ in diameter and the failure process corresponds to that described in Fig. 5(c). 


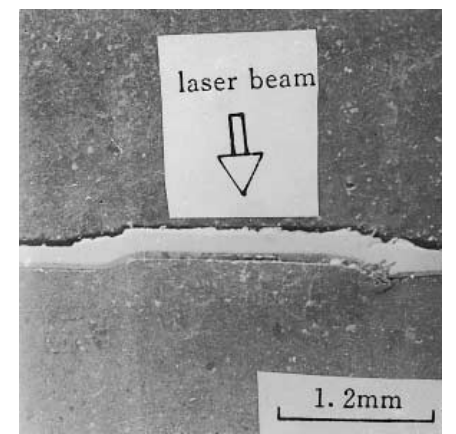

Fig. 6. Photograph of sectioned brass foil at the bulging state with a laser energy of $8.2 \mathrm{~J}$ over an area of $2.3 \mathrm{~mm}$ in diameter.

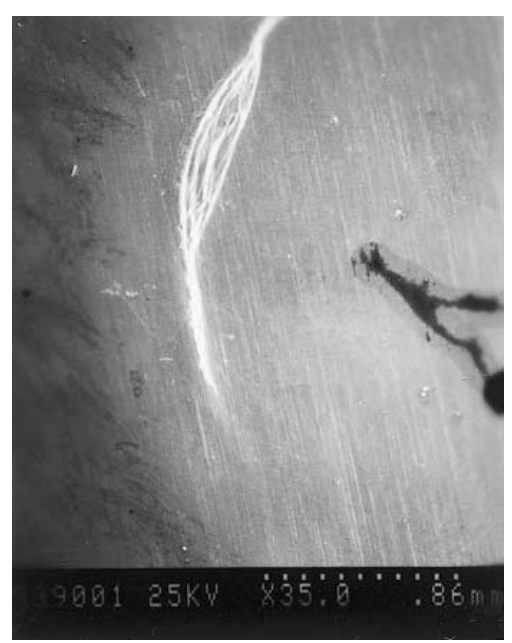

Fig. 7. Photograph of brass foil failure by plugging viewed from the rear surface with a laser energy of $29 \mathrm{~J}$ over a region $4.5 \mathrm{~mm}$ in diameter.

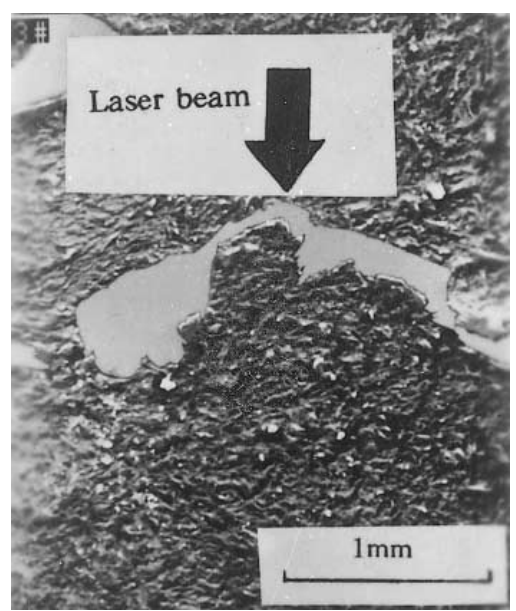

Fig. 8. Photograph of fractured and fragmented brass foil subjected to a laser energy of $2.9 \mathrm{~J}$ over a region $2.3 \mathrm{~mm}$ in diameter. 

When the intensity is increased to $0.75 \times 10^{6} / \mathrm{cm}^{2}$ the brass foil is totally fractured and fragmented. Figure 8 shows a sectioned photograph of fractured and fragmented brass foil subjected to a laser energy of $9.2 \mathrm{~J}$ over a region $2.3 \mathrm{~mm}$ in diameter and the failure process corresponds to that described in Fig. 5(d).

\section{Temperature distributions}

\subsection{Governing equations}

Determination of the temperature fields becomes essential to reveal the mechanism of the newlydiscovered failure node. To find the solution of the temperature fields, we assume: (1) the laser is a unique heat supply to the target material so that no dissipation of irreversible plastic work is contributed to temperature rise; (2) the laser induced thermal deformation is infinitesimal so that the temperature distribution can be solved based on the initial configuration rather than on the deformed ones; (3) thermal parameters of materials are assumed to have no dependence on temperature.

Clearly, under these assumptions, the temperature rise $\theta=T-T_{0}=\theta(r, z, t)$ is decoupled with mechanical deformation, where $T$ and $T_{0}$ are absolute temperature and room temperature, respectively. The temperature rise is described by a two-dimensional heat diffusion equation:

$$
\frac{\partial \theta}{\partial t}=D\left(\frac{\partial^{2} \theta}{\partial r^{2}}+\frac{1}{r} \frac{\partial \theta}{\partial r}+\frac{\partial^{2} \theta}{\partial z^{2}}\right)
$$

with initial and boundary conditions

$$
\begin{aligned}
& \left.\theta\right|_{t=0}=0 \\
& \left.\frac{\partial \theta}{\partial r}\right|_{r=0}=\left.\theta\right|_{r=b}=0 \\
& \left.\frac{\partial \theta}{\partial z}\right|_{z=(h / 2)}=0,\left.\quad \frac{\partial \theta}{\partial z}\right|_{z=-(h / 2)}=-\frac{1}{k}\left(1-R_{0}\right) I_{\max } f(r) g(t)
\end{aligned}
$$

where the functions $g(t)$ and $f(r)$ are given in eqns (1) and (2), respectively. The condition (6) 2 represents the laser absorption by target material on the front surface $z=-h / 2$, where $h$ is the thickness of target, $b$ is the outer radius of sample, $D=k / \rho C_{\mathrm{p}}$ is thermal diffusion coefficient, $R_{0}$ is the reflection coefficient and is equal to 0.9 approximately. Also, $k, \rho$, and $C_{\mathrm{p}}$ are, respectively, thermal conductivity, mass density and specific heat.

For convenience in the subsequent analysis, the dimensionless variables are introduced as follows :

$$
\bar{\theta}=\frac{\theta}{h_{4} T_{m}}, \quad \bar{t}=\frac{D t}{a^{2}}, \quad \bar{r}=\frac{r}{a}, \quad \bar{z}=\frac{z}{a}
$$


where $T_{m}$ is the melting temperature of material. The dimensionless temperature $\bar{\theta}$ depends on the four-dimensionless parameters :

$$
A=\frac{\alpha a^{2}}{D}, \quad B=\frac{(\alpha+\beta) a^{2}}{D}, \quad h_{1}=\frac{h}{a}, \quad h_{4}=\frac{\left(1-R_{0}\right) I_{\max } a}{k T_{m}}
$$

For simplicity, all barred dimensionless variables are supposed to keep their original symbols in the following analysis. The governing equations of temperature fields are expressed in dimensionless forms as follows:

heat-diffusion equation

$$
\frac{\partial \theta}{\partial t}=\frac{\partial^{2} \theta}{\partial r^{2}}+\frac{1}{r} \frac{\partial \theta}{\partial r}+\frac{\partial^{2} \theta}{\partial z^{2}}
$$

with initial and boundary conditions :

$$
\begin{aligned}
& \left.\theta\right|_{t=0}=0 \\
& \left.\frac{\partial \theta}{\partial z}\right|_{z=-\left(h_{1} / 2\right)}=-f(r) g(t),\left.\quad \frac{\partial \theta}{\partial z}\right|_{z=\left(h_{1} / 2\right)}=0,\left.\quad \frac{\partial \theta}{\partial r}\right|_{r=0}=\left.\theta\right|_{r=h_{2}}=0
\end{aligned}
$$

where $h_{2}=b / a$ and the temporal shape $g(t)$ is rewritten in dimensionless form as follows:

$$
g(t)=e^{-A t}-e^{-B t}
$$

Using Hankel transformation and Bessel series expansion technique, the problem (9)-(11) has been solved analytically in Appendix A.

\subsection{Experimental and calculated results}

In this part, we are going to compare experimental data with calculated results for the temperature rise. In order to calculate temperature rise the material parameters need to be known. Table 1 provides a summary of the material constants and laser parameters.

Table 1

Parameters of material and laser beam

\begin{tabular}{lllll}
\hline$\rho\left(\mathrm{g} / \mathrm{cm}^{2}\right)$ & $C_{\mathrm{p}}(\mathrm{J} / \mathrm{g} \mathrm{K})$ & $k(\mathrm{~W} / \mathrm{cm} \mathrm{K})$ & $D\left(\mathrm{~cm}^{2} / \mathrm{s}\right)$ & $\alpha_{0}\left(\mathrm{~K}^{-1}\right)$ \\
\hline 8.7 & 0.374 & 1.09 & 0.335 & $1.7 \times 10^{-5}$ \\
$R_{0}$ & $\sigma_{0}(\mathrm{GPa})$ & $T_{0}(\mathrm{~K})$ & $T_{m}(\mathrm{~K})$ & $\alpha\left(\mathrm{s}^{-1}\right)$ \\
0.9 & 0.20 & 300 & 1356 & $1.5 \times 10^{4}$ \\
$\beta\left(\mathrm{s}^{-1}\right)$ & $h(\mathrm{~mm})$ & $a(\mathrm{~mm})$ & $E(\mathrm{GPa})$ & \\
$8.0 \times 10^{4}$ & 0.1 & 2.5 & 100.0 & \\
\hline
\end{tabular}



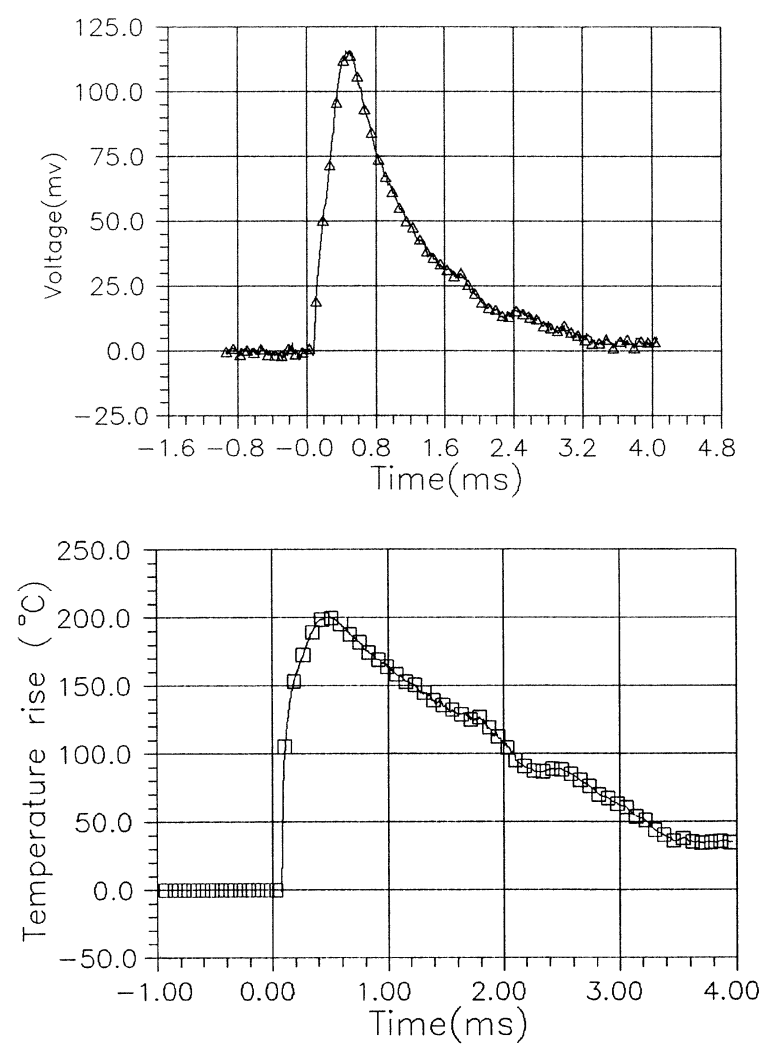

Fig. 9. Histories of the voltage (a) and the corresponding temeperature rise (b) in the center of the rear surface of brass foil, where laser energy and spot diameter are $10.6 \mathrm{~J}$ and $5.2 \mathrm{~mm}$, respectively.

Figure 9(a) shows the voltage history which represents the temperature rise in the center of the rear surface of the sample, where the laser energy and spot diameter are $10.6 \mathrm{~J}$ and $5.2 \mathrm{~mm}$, respectively. It can be seen that the voltage increases from almost zero (from within the noise level of the system) to a maximum value of $115 \mathrm{mv}$ at the time $t=0.4 \mathrm{~ms}$. The rise time of voltage just corresponds to the duration of laser irradiation as shown in Fig. 3. As shown in Fig. 9(b), the temperature rise in the center of the rear surface of brass foil does not happen instantaneously and the slugging time is almost $40 \mu \mathrm{s}$.

Figure 10 shows the temperature rise vs laser energy at $t=0.45 \mathrm{~ms}$, where the recorded signal represents the temperature rise in the center of the rear surface of brass foil. It can be clearly see that temperature rise $\theta$ increase linearly with the laser energy $E_{J}$. This linear dependence of temperature rise on laser energy as well as on the maximum laser intensity $I_{\max }$ is also confirmed by the analysis given in (7) and (8).

Figure 11 shows the histories of the temperature rise in the centers of the front and rear surfaces. The experimental results are also given in the figure. The good agreement of calculated results with the test can be seen in the figure. Figure 12 shows the variations of temperature rise with $z / a$ in the center of brass foil. As expected, a high temperature state is built up on the specimen front surface 


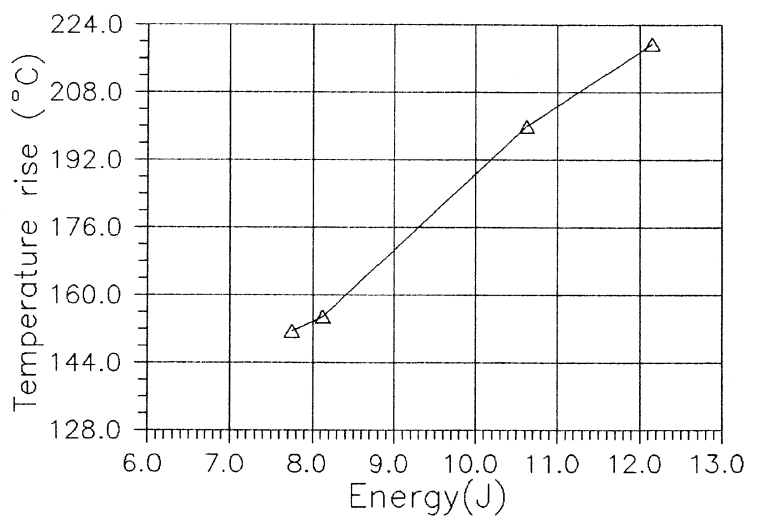

Fig. 10. Temperature rise vs laser energy at $t=0.45 \mathrm{~ms}$, where the recorded signal corresponds to the temperature rise in the center of the rear surface of brass foil.

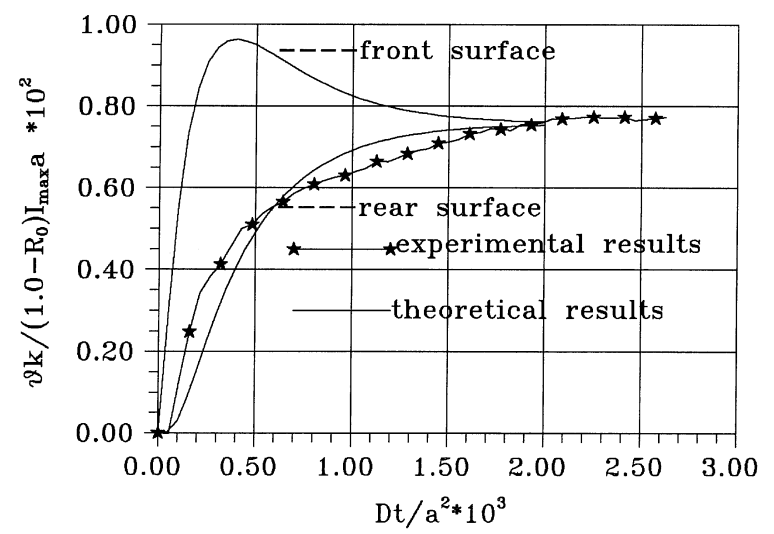

Fig. 11. Histories of the dimensionless temperature rise in the center of laser spot on the front and rear surface.

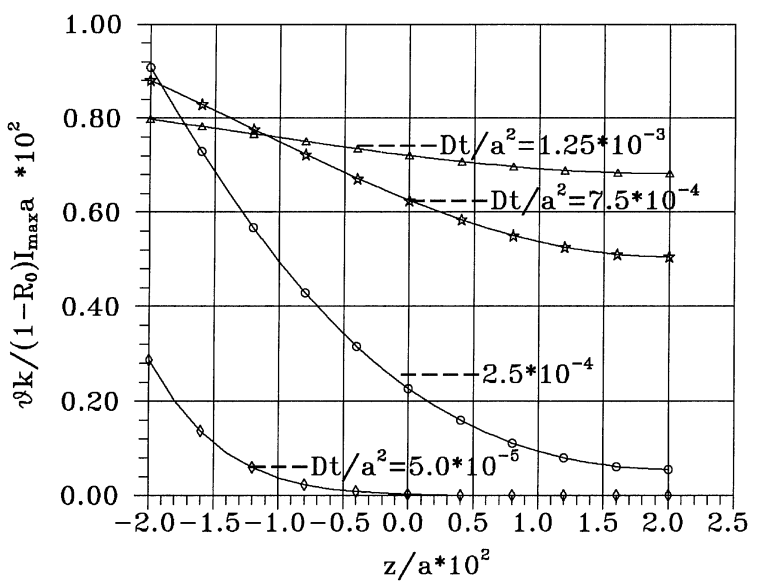

Fig. 12. Variations of dimensionless temeprature rise at different times with $z / a$ in the center of brass foil. 

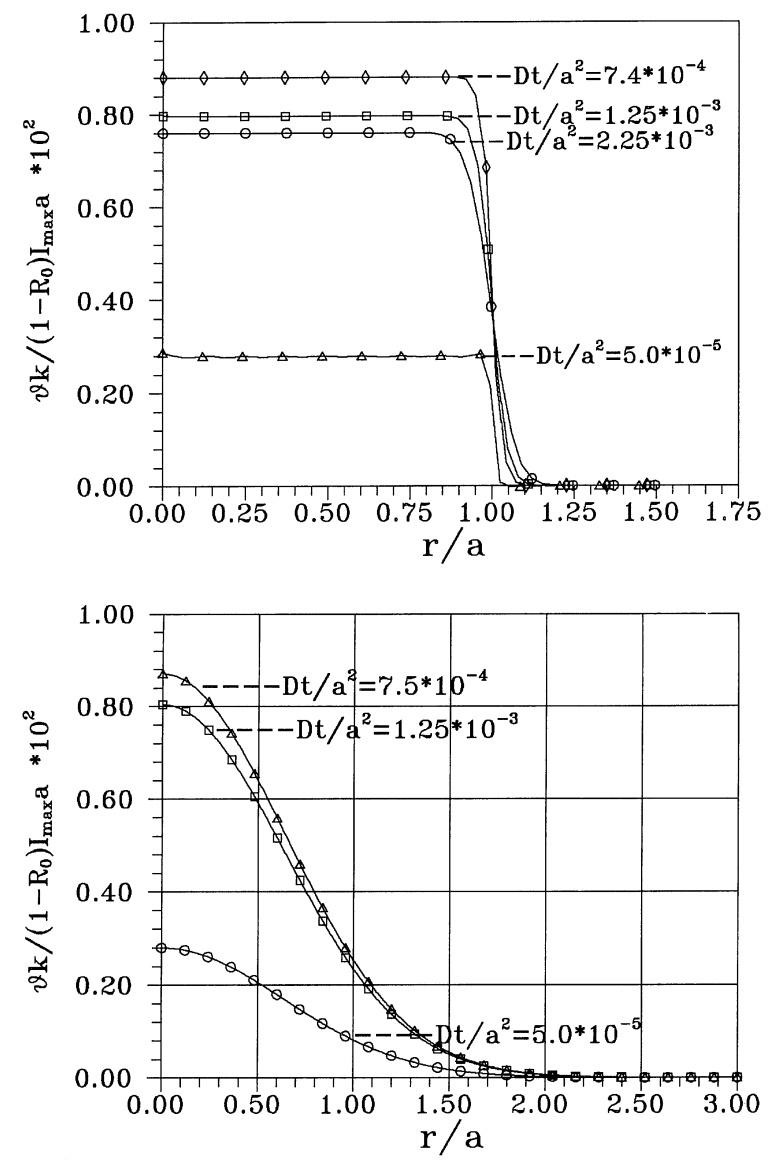

Fig. 13. Variations of non-dimensional temeprature rise with $r / a$ on the front surface at different time, where the spatial shape of laser is non-Gaussian (a) or Gaussian (b) type.

impinged by a normal incident laser beam. This means that the temperature gradient in $z$-direction, i.e., $\partial \theta / \partial z$ is high at the earlier stage of laser irradiation, and then gradually decays. At the late stage of laser irradiation, the temperature gradient tends to zero. As indicated in the following section, this temperature gradient plays a key role in leading to the brass foil bulging.

The spatial profile of temperature distribution is influenced definitely by the spatial shape of the laser beam, as shown in Fig. 13(a)-(b). Therefore, different spatial shapes of laser beam lead to different temperature distributions even though the net energy and duration are the same. For non-Gaussian type laser as used in the test, the spatial distribution of the temperature is uniform within the laser irradiated region and declines very sharply towards the edge where the laser spot terminates. The steep temperature gradient concentrates across the periphery of the laser where the spot terminates. As we can see in the following section or in Zhou et al. (1998), this causes high shear deformation to occur around a rim near the outer edge of the laser beam. 


\section{Thermal bulging}

\subsection{Governing equations}

As shown in Section 2, at the earlier stage of laser irradiations, the brass foil bulges towards the side of the incident laser beam. The classical Kirchhoff plate theory can be used to determine the bulging, i.e., the deflection $w=w(r, t)$. The assumption is reasonable that the deformation is infinitesimal so that the membrane and shear forces can be neglected. Thus, the governing equation for $w$ is given by (Boley and Weiner, 1960)

$$
D_{1} \nabla^{4} w+\frac{1}{1-v} \nabla^{2} M_{T}+\rho h \ddot{w}=0
$$

where $D_{1}=\left[E h^{3} /\left(12\left(1-v^{2}\right)\right)\right]$ denotes the bending stiffness, and $E, v$ and $\alpha_{0}$ are, respectively, Young's modulus, Poisson ratio and the thermal expansion coefficient of material. The corresponding initial, boundary and symmetrical conditions are

$$
\left\{\begin{array}{l}
\left.w\right|_{t=0}=\left.\dot{w}\right|_{t=0}=0 \\
\left.w\right|_{r=b}=\left.\frac{\partial w}{\partial r}\right|_{r=b}=0,\left.\quad w\right|_{r=0}=\text { finite, }\left.\quad \frac{\partial w}{\partial r}\right|_{r=0}=\text { finite }
\end{array}\right.
$$

In eqn (13), the equivalent thermal bending moment $M_{T}$ is given by

$$
M_{T}=\alpha_{0} E \int_{-h / 2}^{h / 2} \theta(r, z, t) z \mathrm{~d} z
$$

and the equivalent transverse loading $q_{\theta}$ can be defined as

$$
q_{\theta}=-\frac{1}{1-v} \nabla^{2} M_{T}=-\frac{\alpha_{0} E}{1-v} \int_{-h / 2}^{h / 2} z \nabla^{2} \theta \mathrm{d} z
$$

where $\nabla^{2}=\left(\mathrm{d}^{2} / \mathrm{d} r^{2}\right)+(1 / r)(\mathrm{d} / \mathrm{d} r)$ is the Laplace operator given in axisymmetrical coordinate system, schemed in Fig. 1. Since the solution for temperature rise $\theta=\theta(r, z, t)$ has already been obtained in (A.22), the thermal bending moment $M_{T}$ and the equivalent transverse loading $q_{\theta}$ are entirely known before the eqns (13) with (14) are solved for $w$.

For convenience in subsequent analysis, the dimensionless variables are introduced in the forms :

$$
\bar{w}=\frac{w}{W_{p} a}, \quad \bar{M}_{T}=\frac{M_{T}}{M_{p} M_{0}^{0}}, \quad \bar{q}_{\theta}=\frac{q_{\theta}}{\sigma_{0} M_{p}}
$$

where

$$
W_{p}=24(1+v) \alpha_{0} \frac{\left(1-R_{0}\right) a I_{\max }}{k}, \quad M_{p}=8 \alpha_{0} \frac{\left(1-R_{0}\right) a I_{\max }}{k} \frac{E}{\sigma_{0}}, \quad M_{0}^{0}=\frac{1}{4} h^{2} \sigma_{0}
$$

and $\sigma_{0}$ is yield strength of material. The material constants and laser parameters are listed in Table 1. For simplicity, all barred dimensionless variables are supposed to keep their original symbols in the following derivation. The other dimensionless variables, such as $\theta, t, r$ and $z$ keep the same as 
in (7). Using the solution (A.22) for $\theta$ and the formulae (15)-(16), the dimensionless thermal bending moment $M_{T}$ and the equivalent transverse loading $q_{\theta}$ can be easily obtained as follows,

$$
\begin{aligned}
& M_{T}=\frac{1}{2 h_{1}^{2}} \int_{-h_{1} / 2}^{h_{1} / 2} \theta z \mathrm{~d} z=-\frac{h_{1}}{h_{2}^{2}} \sum_{k_{n}} \frac{J_{0}\left(k_{n} r\right) f^{*}\left(k_{n}\right)}{J_{1}^{2}\left(k_{n} h_{2}\right)} \bar{g}\left(k_{n}, t\right) \\
& q_{\theta}=-\frac{1}{1-v} \frac{h_{1}^{2}}{4} \nabla^{2} M_{T}=-\frac{h_{1}}{h_{2}^{2}} \frac{1}{4(1-v)} \sum_{k_{n}} \frac{k_{n} J_{0}\left(k_{n} r\right) f^{*}\left(k_{n}\right)}{J_{1}^{2}\left(k_{n} h_{2}\right)} \bar{g}\left(k_{n}, t\right)
\end{aligned}
$$

where $J_{0}(x)$ and $J_{1}(x)$ are, respectively, the zeroth and first Bessel functions, and

$$
\bar{g}\left(k_{n}, t\right)=\frac{1}{24} g(t)+2 \sum_{m=1}^{\infty} \frac{\left[(-1)^{m}-1\right]}{(m \pi)^{4}}\left[g(t)-\left(\frac{m \pi}{h_{1}}\right)^{2}\left(\frac{e^{-A t}-e^{-C t}}{C-A}-\frac{e^{-B t}-e^{-C t}}{C-B}\right)\right]
$$

To solve eqn (13) with the prescribed conditions (14), we are able to decompose $w$ into two parts (Das, 1983; Tauchert, 1989), the quasi-static part $w_{s}$ and the dynamic part $w_{d}$

$$
w=w_{s}+w_{d}
$$

and they satisfy, respectively, the following equations :

$$
\nabla^{4} w_{s}=-\frac{1}{h_{1}} \nabla^{2} M_{T}, \quad \nabla^{4} w_{d}+m_{1} \ddot{w}_{d}=-m_{1} \ddot{w}_{s}
$$

where $m_{1}=12\left(1-v^{2}\right) \rho D^{2} / E h^{2}$. The corresponding initial and boundary conditions are

$$
\left.w_{d}\right|_{t=0}=-\left.w_{s}\right|_{t=0},\left.\quad \dot{w}_{d}\right|_{t=0}=-\left.\dot{w}_{s}\right|_{t=0}
$$

and

$$
\left\{\begin{array}{l}
\left.w_{s}\right|_{r=0}=\text { finite },\left.\quad \frac{\partial w_{s}}{\partial r}\right|_{r=0}=\text { finite, }\left.\quad w_{s}\right|_{r=h_{2}}=\left.\frac{\partial w_{s}}{\partial r}\right|_{r=h_{2}}=0 \\
\left.w_{d}\right|_{r=0}=\text { finite, }\left.\quad \frac{\partial w_{d}}{\partial r}\right|_{r=0}=\text { finite, }\left.\quad w_{d}\right|_{r=h_{2}}=\left.\frac{\partial w_{d}}{\partial r}\right|_{r=h_{2}}=0
\end{array}\right.
$$

These solutions for $w_{s}$ and $w_{\mathrm{d}}$ can be obtained by using a series expansion technique. The quasistatic deflection $w_{s}$ can be easily obtained as follows:

$$
w_{s}=-\frac{1}{h_{2}^{2}} \sum_{k_{n}} \frac{f^{*}\left(k_{n}\right) \bar{g}\left(k_{n}, t\right)}{k_{n}^{2} J_{1}^{2}\left(k_{n} h_{2}\right)}\left[J_{0}\left(k_{n} r\right)+\frac{1}{2 h_{2}} k_{n} J_{1}\left(k_{n} h_{2}\right)\left(r^{2}-h_{2}^{2}\right)\right]
$$

The derivation of dynamical deflection $w_{d}$ is rather laborious and it is given in Appendix B. 

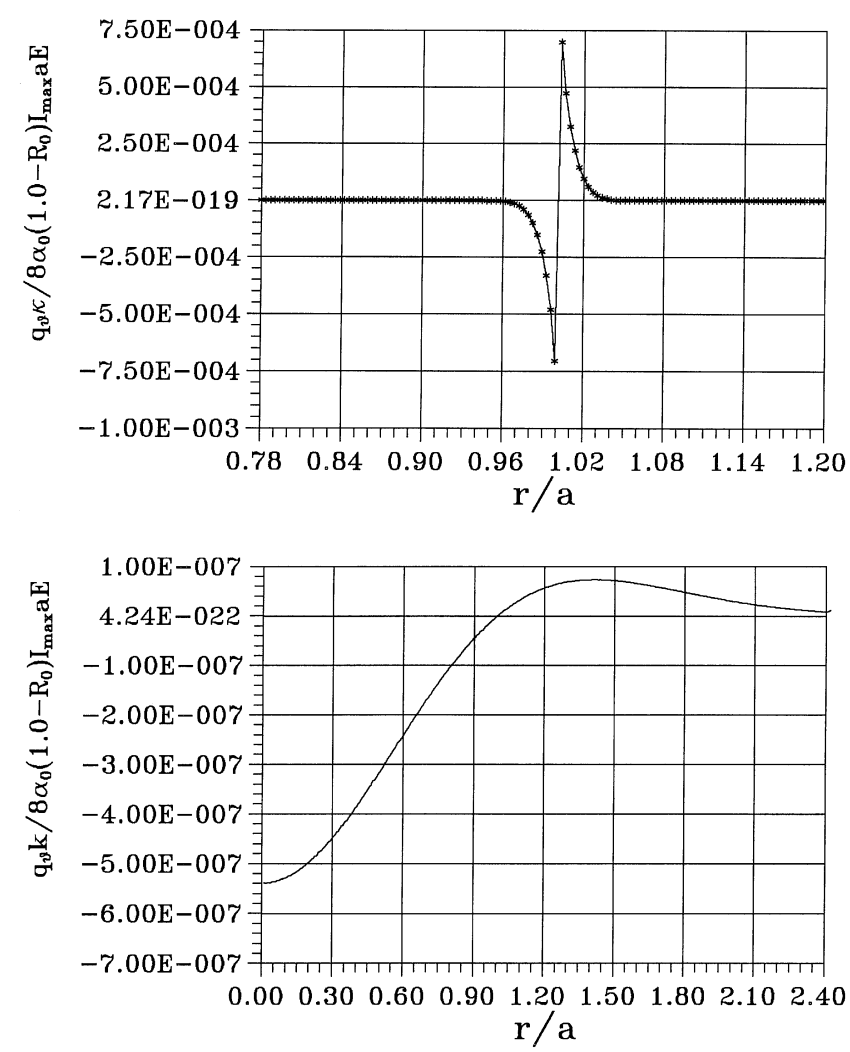

Fig. 14. Spatial distribution of equivalent transverse loading $q_{\theta}$ induced by non-Gaussian laser beam (a) and Gaussian laser beam (b).

\subsection{Calculated results}

\subsubsection{Equivalent transverse loading $\mathrm{q}_{\theta}$}

The numerical computations for the equivalent transverse loading $q_{\theta}$ are schematically shown in Fig. 14(a)-(b) in the cases of Gaussian and non-Gaussian laser beam. The spatial profile of temperature rise or the thermal moment $M_{T}$ is almost the same as that of laser beam. This causes that $q_{\theta}$ is constantly negative in the irradiated region and is constantly positive near the outer edge of the laser beam. However, in the case of metal projectiles penetrating through metal targets in plate form, the transverse loading is constantly positive and zero within and around the projectiles penetrating region, respectively. The negative value of $q_{\theta}$ within the irradiated region causes the foil to bulge towards the direction opposite to laser irradiation. The spatial profiles of $q_{\theta}$ for different spatial shapes of laser beam are evidently different. The equivalent transverse loading $q_{\theta}$ varies with $r / a$ in the peripheral region much more sharply in the non-Gaussian type of laser beam than that in the Gaussian type of laser beam. The distinct feature of the spatial distribution of the laser intensity, or the equivalent transverse loading $q_{\theta}$ causes the shear strain at the periphery of the laser spot is much larger than that in other regions (Zhou et al., 1998). High shear deformation concentrated in a rim is developed and causes a circular region impinged upon by the laser to 

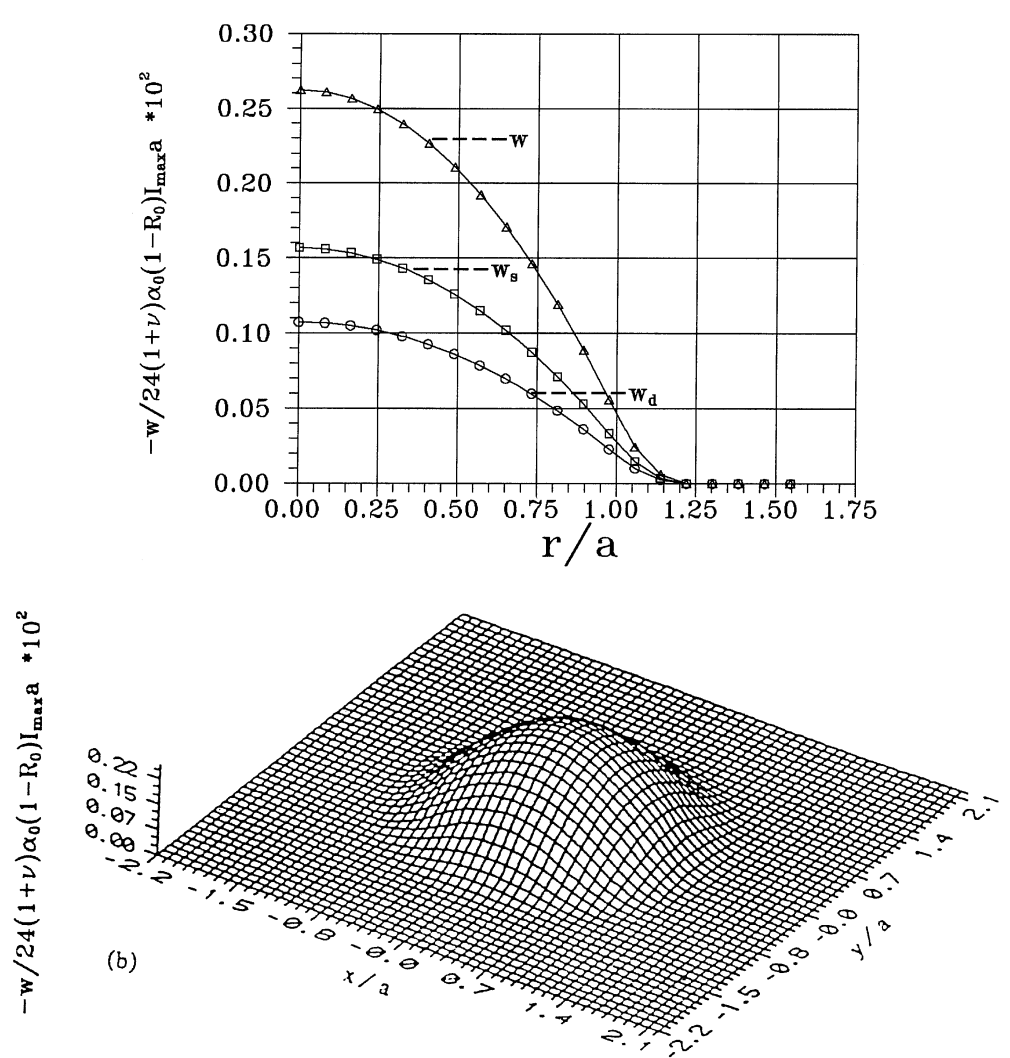

Fig. 15. Deflection curve for the brass foil: (a) two-dimensional profile; (b) three-dimensional profile, where $D t / a^{2}=3.0 \times 10^{-4}$.

move towards bulging direction. From the comparison of equivalent transverse loading $q_{\theta}$ (Fig. 14) or the shear distributions (Zhou et al., 1998) induced by non-Gaussian type laser beam with that induced by Gaussian type laser beam, one can conclude that the former offers a formidable potential for the new type of failure by plugging, however, the latter has a litter potential for the new type of failure by plugging. This reveals that the spatial shapes of laser beams play an important role in controlling the damage types.

\subsubsection{Deflection}

The deflections for $w, w_{s}$ and $w_{d}$ are, respectively, depicted in Fig. 15(a)-(b). It is again confirmed from these figures that the brass foil truly bulges towards opposite to the direction of laser beam. As shown in Fig. 16 and Fig. 17, the contribution of $w_{s}$ to bulging is always positive and $w_{d}$ reveals a flexural vibration in a long period of time. Meanwhile, the temperature profiles and equivalent transverse loading $q_{\theta}$ vary much more abruptly with $r$ in the laser spot edge for the non-Gaussian laser beam than for the Gaussian laser beam, so do the curvature of deflection curves. On the other hand, the thermal stress in the laser spot edge region has already exceeded the corresponding yield stress under the elevated temperature condition. In this case, the classical thermal-elastic decoupling 


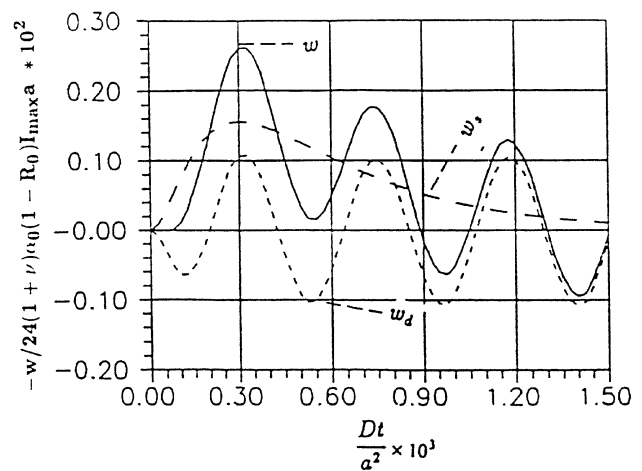

Fig. 16. Histories of $w_{s}, w_{d}$ and $w$ for the center of brass foil.

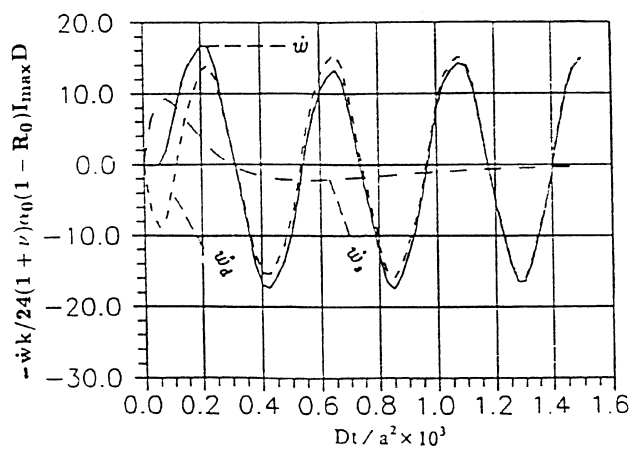

Fig. 17. Histories of velocity $\dot{w}$ in the center of brass foil.

plate theory could no longer be satisfactory in analyzing the stress and strain distributions within this very narrow region. Therefore, developing a thermal-elastic plastic coupling theory for the study of thermal shear stress concentration in the laser spot edge region plays actually an essential role in understanding the new failure mode at the second stage of laser irradiation.

\section{Concluding remarks}

A new kind of laser-induced failure mode has been reported experimentally. This newly-discovered phenomenon is different from the well-known types of laser induced material damages, such as spallation, melting and vaporization. The observed plugging failure can be summarized in three stages. They are referred to as reverse bulging, localized shear deformation and perforation by plugging. In order to study this newly-discovered phenomenon, an analytical solution for twodimensional axisymmetrical temperature distribution is obtained in terms of Hankel transformation and series expansion techniques. Using this temperature solution and the classical Kirchhoff plate theory, deflection curve, i.e., reverse bulging $w$ can be determined analytically under the assumption of thermal-mechanical decoupling. These analyses reveal that: (a) the 
thermal reverse bulging is mainly due to highly nonuniform spatial distribution of temperature in normal direction of target material, i.e., the temperature gradient in $z$-direction; (b) a steep temperature gradient across the periphery of the laser spot causes the shear strain at the periphery of the laser spot to be much larger than that in other regions. It is the temperature rise and subsequent shear deformation localization which control the whole process of the new failure mode. In conclusion, our investigation on the new failure mode reveals that in addition to the incident laser intensity and duration, the spatial structure of the laser beam also plays an important role in controling the damage.

\section{Acknowledgement}

Support for this research program was provided partly by NNSF of China and partly by the Field of Laser Technology, 863-NHT Research Development Program. This support is gratefully acknowledged.

\section{Appendix A}

In this appendix, the temperature distribution governed by eqns (9)-(11) is analytically solved. To do this, let us first consider the boundary condition (11) and assume the solution of eqn (9) to be series expansion form

$$
\theta=\sum_{k_{n}} \theta^{*}\left(k_{n}, z, t\right) J_{0}\left(k_{n} r\right)
$$

where $J_{n}(x)$ are the $n$-th Bessel functions of the first kind and $k_{n}$ are the roots of equation $J_{0}\left(k_{n} h_{2}\right)=0$. Substituting the right-hand side of the above expression into the original equation (9) as well as the initial and boundary conditions (10)-(11), we obtain

$$
\begin{aligned}
& \frac{\partial \theta^{*}}{\partial t}=-k_{n}^{2} \theta^{*}+\frac{\partial^{2} \theta^{*}}{\partial z^{2}} \\
& \left.\theta^{*}\right|_{t=0}=0 \\
& -\left.\frac{\partial \theta^{*}}{\partial z}\right|_{z=-\left(h_{1} / 2\right)}=\frac{2 f^{*}\left(k_{n}\right) g(t)}{h_{2}^{2} J_{1}^{2}\left(k_{n} h_{2}\right)},\left.\quad \frac{\partial \theta^{*}}{\partial z}\right|_{z=\left(h_{1} / 2\right)}=0
\end{aligned}
$$

where $f^{*}\left(k_{n}\right)$ are called the transforming coefficient and are given by

$$
f^{*}\left(k_{n}\right)=\int_{0}^{h_{2}} f(r) J_{0}\left(k_{n} r\right) r \mathrm{~d} r
$$

If we take $f(r)=e^{-r^{2}}$ in (A.5), we may obtain 


$$
f^{*}\left(k_{n}\right)=\frac{J_{1}\left(k_{n}\right)}{k_{n}}
$$

By substituting expression (2) into (A.5), we may obtain the transforming coefficients

$$
f^{*}\left(k_{n}\right)=\sum_{l=0}^{\infty} \frac{(-1)^{l}}{l !} \frac{Q_{l}}{k_{n}^{2 l+2}}
$$

where

$$
Q_{0}=h_{2} k_{n} J_{1}\left(k_{n} h_{2}\right), \quad Q_{l}=\left(h_{2} k_{n}\right)^{2 l+1} J_{1}\left(k_{n} h_{2}\right)-4 l^{2} Q_{l-1} \quad(l=1,2, \ldots)
$$

Considering the boundary condition (A.4), we introduce a new function $V^{*}$ to replace $\theta^{*}$ in the form

$$
\theta^{*}\left(k_{n}, z, t\right)=\psi g(t)\left(z-\frac{h_{1}}{2}\right)^{2}+V^{*}\left(k_{n}, z, t\right)
$$

where

$$
\psi=\frac{f^{*}\left(k_{n}\right)}{h_{1} h_{2}^{2} J_{1}^{2}\left(k_{n} h_{2}\right)}
$$

and then eqns (A.2)-(A.4) may be converted into the form

$$
\begin{aligned}
& \frac{\partial V^{*}}{\partial t}=\frac{\partial^{2} V^{*}}{\partial z^{2}}-k_{n}^{2} V^{*}+2 \psi g(t)-\psi\left[\dot{g}(t)+k_{n}^{2} g(t)\right]\left(z-\frac{h_{1}}{2}\right)^{2} \\
& \left.\frac{\partial V^{*}}{\partial z}\right|_{z= \pm\left(h_{1} / 2\right)}=0 \\
& \left.V^{*}\right|_{t=0}=0
\end{aligned}
$$

Now, let us expand $V^{*}\left(k_{n}, z, t\right)$ in terms of cosine series as follows:

$$
V^{*}\left(k_{n}, z, t\right)=\sum_{m=0}^{\infty} A_{m} \cos \left[\frac{\pi m}{h_{1}}\left(z+\frac{h_{1}}{2}\right)\right]
$$

where the unknown variables $A_{m}$ satisfy

$$
\begin{aligned}
& \dot{A}_{m}+\left[\left(\frac{m \pi}{h_{1}}\right)^{2}+k_{n}^{2}\right] A_{m}=\Phi_{m}^{*} \\
& \left.A_{m}\right|_{t=0}=0
\end{aligned}
$$

In eqn (15), 


$$
\Phi_{0}^{*}=\frac{1}{h_{1}} \int_{-h_{1} / 2}^{h_{1} / 2}\left\{2 g(t)-\left[\dot{g}(t)+k_{n}^{2} g(t)\right]\left(z-\frac{h_{1}}{2}\right)^{2}\right\} \psi \mathrm{d} z
$$

and

$$
\Phi_{m}^{*}=\frac{2}{h_{1}} \int_{-h_{1} / 2}^{h_{1} / 2}\left\{2 g(t)-\left[\dot{g}(t)+k_{n}^{2} g(t)\right]\left(z-\frac{h_{1}}{2}\right)^{2}\right\} \psi \cos \left[\frac{m \pi}{h_{1}}\left(z+\frac{h_{1}}{2}\right)\right] \mathrm{d} z
$$

By substituting (12) into (A.17)-(A.18), we obtain

$$
\begin{aligned}
& A_{0}=2 \psi\left[\frac{e^{-A t}-e^{-k_{n}^{2} t}}{k_{n}^{2}-A}-\frac{e^{-B t}-e^{-k_{n}^{2} t}}{k_{n}^{2}-B}-\frac{1}{6} h_{1}^{2} g(t)\right], \quad(m=0) \\
& A_{m}=-\psi\left(\frac{2 h_{1}}{m \pi}\right)^{2}\left\{g(t)-\left(\frac{m \pi}{h_{1}}\right)^{2}\left[\frac{e^{-A t}-e^{-C t}}{C-A}-\frac{e^{-B t}-e^{-C t}}{C-B}\right]\right\}, \quad(m=1,2, \ldots)
\end{aligned}
$$

where

$$
C=k_{n}^{2}+\left(\frac{m \pi}{h_{1}}\right)^{2}
$$

Finally, the exact solution of temperature distributions is given below

$$
\begin{aligned}
\theta=\sum_{k_{n}} & \frac{2 J_{0}\left(k_{n} r\right) f^{*}\left(k_{n}\right)}{h_{1} h_{2}^{2} J_{1}^{2}\left(k_{n} h_{2}\right)}\left\{\frac{1}{2} g(t)\left(z-\frac{h_{1}}{2}\right)^{2}-\frac{1}{6} h_{1}^{2} g(t)+\frac{e^{-A t}-e^{-k_{n}^{2} t}}{k_{n}^{2}-A}-\frac{e^{-B t}-e^{-k_{n}^{2} t}}{k_{n}^{2}-B}\right. \\
& \left.-\sum_{m=1}^{\infty} \frac{2 h_{1}^{2}}{(m \pi)^{2}}\left[g(t)-\left(\frac{m \pi}{h_{1}}\right)^{2}\left(\frac{e^{-A t}-e^{-C t}}{C-A}-\frac{e^{-B t}-e^{-C t}}{C-B}\right)\right] \cos \left[\frac{m \pi}{h_{1}}\left(z+\frac{h_{1}}{2}\right)\right]\right\}
\end{aligned}
$$

Thus, as long as the roots $k_{n}$ are known, the solution for $\theta$ is entirely obtained.

\section{Appendix B}

To obtain the exact solution for $w_{d}$ in eqn (23) together with the prescribed conditions (24) and (25), we assume that the dynamic deflection $w_{d}$ is expanded in the form:

$$
w_{d}=\sum_{\alpha_{n}} w_{d}^{*}\left(\alpha_{n}, t\right)\left[I_{0}\left(\alpha_{n} r\right)-\frac{I_{0}\left(\alpha_{n} h_{2}\right)}{J_{0}\left(\alpha_{n} h_{2}\right)} J_{0}\left(\alpha_{n} r\right)\right]
$$

where $\alpha_{n}$ are the roots of the following equation:

$$
J_{0}\left(\alpha_{n} h_{2}\right) I_{1}\left(\alpha_{n} h_{2}\right)+J_{1}\left(\alpha_{n} h_{2}\right) I_{0}\left(\alpha_{n} h_{2}\right)=0
$$

$J_{n}(x)$ and $I_{n}(x)(n=0,1)$ are, respectively, the $n$-th Bessel function and the $n$-th modified Bessel function. Let $y_{n}$ be 


$$
y_{n}=I_{0}\left(\alpha_{n} r\right)-\frac{I_{0}\left(\alpha_{n} h_{2}\right)}{J_{0}\left(\alpha_{n} h_{2}\right)} J_{0}\left(\alpha_{n} r\right)
$$

we may readily prove that the eigenfunctions $y_{n}$ are complete and orthogonal, that is,

$$
\int_{0}^{h_{2}} y_{m} y_{n} r \mathrm{~d} r=0, \quad(m \neq n)
$$

In order to solve exactly the dynamic deflection $w_{d}$, some modules and transforming coefficients should be derived below.

\section{A. Derivation of modules $\int_{0}^{\mathrm{h}_{2}} \mathrm{y}_{\mathrm{n}}^{2} \mathrm{r} d \mathrm{r}$}

Substituting (B.3) into $\int_{0}^{h_{2}} y_{n}^{2} r \mathrm{~d} r$, we obtain

$$
\int_{0}^{h_{2}} y_{n}^{2} r \mathrm{~d} r=\int_{0}^{h_{2}}\left[I_{0}^{2}\left(\alpha_{n} r\right)+\frac{I_{0}^{2}\left(\alpha_{n} h_{2}\right)}{J_{0}^{2}\left(\alpha_{n} h_{2}\right)} J_{0}^{2}\left(\alpha_{n} r\right)-\frac{2 I_{0}\left(\alpha_{n} h_{2}\right)}{J_{0}\left(\alpha_{n} h_{2}\right)} I_{0}\left(\alpha_{n} r\right) J_{0}\left(\alpha_{n} r\right)\right] r \mathrm{~d} r
$$

Considering the following expression

$$
\int_{0}^{h_{2}} I_{0}^{2}\left(\alpha_{n} r\right) r \mathrm{~d} r=\frac{1}{2} h_{2}^{2} I_{0}^{2}\left(\alpha_{n} h_{n}\right)-\int_{0}^{h_{2}} r^{2} I_{0}\left(\alpha_{n} r\right) \frac{\mathrm{d} I_{0}\left(\alpha_{n} r\right)}{\mathrm{d} r} \mathrm{~d} r
$$

and the modified Bessel equation,

$$
\frac{\mathrm{d}^{2} I_{0}\left(\alpha_{n} r\right)}{\mathrm{d} r^{2}}+\frac{1}{r} \frac{\mathrm{d} I_{0}\left(\alpha_{n} r\right)}{\mathrm{d} r}-\alpha_{n}^{2} I_{0}\left(\alpha_{n} r\right)=0
$$

we have

$$
\int_{0}^{h_{2}} I_{0}^{2}\left(\alpha_{n} r\right) r \mathrm{~d} r=\frac{1}{2} h_{2}^{2}\left[I_{0}^{2}\left(\alpha_{n} h_{2}\right)-I_{1}^{2}\left(\alpha_{n} h_{2}\right)\right]
$$

Similarly, utilizing the Bessel equation

$$
r^{2} \frac{\mathrm{d}^{2} J_{0}\left(\alpha_{n} r\right)}{\mathrm{d} r^{2}}+r \frac{\mathrm{d} J_{0}\left(\alpha_{n} r\right)}{\mathrm{d} r}+\alpha_{n}^{2} r^{2} J_{0}\left(\alpha_{n} r\right)=0
$$

we obtain easily

$$
\int_{0}^{h_{2}} J_{0}^{2}\left(\alpha_{n} r\right) r \mathrm{~d} r=\frac{1}{2} h_{2}^{2}\left[J_{0}^{2}\left(\alpha_{n} h_{2}\right)+J_{1}^{2}\left(\alpha_{n} h_{2}\right)\right], \quad \int_{0}^{h_{2}} I_{0}\left(\alpha_{n} r\right) J_{0}\left(\alpha_{n} r\right) r \mathrm{~d} r=0
$$

Finally, (B.5) becomes 


$$
\int_{0}^{h_{2}} y_{n}^{2} r \mathrm{~d} r=h_{2}^{2} I_{0}^{2}\left(\alpha_{n} h_{2}\right)
$$

\section{B. Calculation of transforming coefficients}

(1) $\int_{0}^{h_{2}} J_{0}\left(k_{n} r\right) y_{n}\left(\alpha_{n} r\right) r \mathrm{~d} r$

Using (B.3), we have

$$
\int_{0}^{h_{2}} J_{0}\left(k_{n} r\right) y_{n}\left(\alpha_{n} r\right) r \mathrm{~d} r=\int_{0}^{h_{2}} J_{0}\left(k_{n} r\right)\left[I_{0}\left(\alpha_{n} r\right)-\frac{I_{0}\left(\alpha_{n} h_{2}\right)}{J_{0}\left(\alpha_{n} h_{2}\right)} J_{0}\left(\alpha_{n} r\right)\right] r \mathrm{~d} r
$$

Utilizing the Bessel eqn (B.7) and the following equation

$$
r^{2} \frac{\mathrm{d}^{2} J_{0}\left(k_{n} r\right)}{\mathrm{d} r^{2}}+r \frac{\mathrm{d} J_{0}\left(k_{n} r\right)}{\mathrm{d} r}+k_{n}^{2} r^{2} J_{0}\left(k_{n} r\right)=0
$$

we have

$$
-\left(k_{n}^{2}+\alpha_{n}^{2}\right) r I_{0}\left(\alpha_{n} r\right) J_{0}\left(k_{n} r\right)=\frac{\mathrm{d}}{\mathrm{d} r}\left[r I_{0}\left(\alpha_{n} r\right) \frac{\mathrm{d} J_{0}\left(k_{n} r\right)}{\mathrm{d} r}-r \frac{\mathrm{d} I_{0}\left(\alpha_{n} r\right)}{\mathrm{d} r} J_{0}\left(k_{n} r\right)\right]
$$

Consequently, we have

$$
\int_{0}^{h_{2}} r I_{0}\left(\alpha_{n} r\right) J_{0}\left(k_{n} r\right) \mathrm{d} r=\frac{h_{2} k_{n}}{k_{n}^{2}+\alpha_{n}^{2}} I_{0}\left(\alpha_{n} h_{2}\right) J_{1}\left(k_{n} h_{2}\right)
$$

By a similar procedure, the following expression is derived

$$
\int_{0}^{h_{2}} r J_{0}\left(\alpha_{n} r\right) J_{0}\left(k_{n} r\right) \mathrm{d} r=\frac{h_{2} k_{n}}{k_{n}^{2}-\alpha_{n}^{2}} J_{0}\left(\alpha_{n} h_{2}\right) J_{1}\left(k_{n} h_{2}\right)
$$

Finally, we calculate the transforming coefficient as

$$
\int_{0}^{h_{2}} J_{0}\left(k_{n} r\right) y_{n}\left(\alpha_{n} r\right) r \mathrm{~d} r=-\frac{2 h_{2} k_{n} \alpha_{n}^{2}}{k_{n}^{4}-\alpha_{n}^{4}} I_{0}\left(\alpha_{n} h_{2}\right) J_{1}\left(k_{n} h_{2}\right)
$$

(2) $\int_{0}^{h_{2}}\left(h_{2}^{2}-r^{2}\right) y_{n}\left(\alpha_{n} r\right) r \mathrm{~d} r$

Considering the following expressions

$$
\int_{0}^{h_{2}} I_{0}\left(\alpha_{n} r\right) r \mathrm{~d} r=\frac{h_{2}}{\alpha_{n}} I_{1}\left(\alpha_{n} h_{2}\right), \quad \int_{0}^{h_{2}} J_{0}\left(\alpha_{n} r\right) r \mathrm{~d} r=\frac{h_{2}}{\alpha_{n}} J_{1}\left(\alpha_{n} h_{2}\right)
$$

we have

$$
\int_{0}^{h_{2}} y_{n}\left(\alpha_{n} r\right) r \mathrm{~d} r=\frac{2 h_{2}}{\alpha_{n}} I_{1}\left(\alpha_{n} h_{2}\right)
$$




$$
\begin{aligned}
& \int_{0}^{h_{2}} I_{0}\left(\alpha_{n} r\right) r^{3} \mathrm{~d} r=\frac{h_{2}^{3}}{\alpha_{n}} I_{1}\left(\alpha_{n} h_{2}\right)-\frac{2 h_{2}^{2}}{\alpha_{n}^{2}} I_{0}\left(\alpha_{n} h_{2}\right)+\frac{4 h_{2}}{\alpha_{n}^{3}} I_{1}\left(\alpha_{n} h_{2}\right) \\
& \int_{0}^{h_{2}} J_{0}\left(\alpha_{n} r\right) r^{3} \mathrm{~d} r=\frac{h_{2}^{3}}{\alpha_{n}} J_{1}\left(\alpha_{n} h_{2}\right)+\frac{2 h_{2}^{2}}{\alpha_{n}^{2}} J_{0}\left(\alpha_{n} h_{2}\right)-\frac{4 h_{2}}{\alpha_{n}^{3}} J_{1}\left(\alpha_{n} h_{2}\right)
\end{aligned}
$$

consequently, we have

$$
\int_{0}^{h_{2}}\left(h_{2}^{2}-r^{2}\right) y_{n}\left(\alpha_{n} r\right) r \mathrm{~d} r=\frac{4 h_{2}^{2}}{\alpha_{n}^{2}} I_{0}\left(\alpha_{n} h_{2}\right)
$$

\section{Transforming coefficient $w_{\mathrm{d}}^{*}\left(\alpha_{\mathrm{n}}, \mathrm{t}\right)$}

Substituting (B.1) into eqn (23), we find

$$
\sum_{\alpha_{n}} \alpha_{n}^{4} w_{d}^{*}\left(\alpha_{n}, t\right) y_{n}\left(\alpha_{n} r\right)+m_{1} \sum_{\alpha_{n}} \ddot{w}_{d}^{*}\left(\alpha_{n}, t\right) y_{n}\left(\alpha_{n} r\right)+m_{1} \ddot{w}_{s}=0
$$

Considering the orthogonality of eigenfunctions $y_{n}$, the right hand side and the left hand side of eqn (B.23) multiplied by $y_{n}\left(\alpha_{n} r\right) r$ simultaneously and integrating them, we obtain the following differential equation

$$
\alpha_{n}^{4} h_{2}^{2} I_{0}^{2}\left(\alpha_{n} h_{2}\right) w_{d}^{*}\left(\alpha_{n}, t\right)+m_{1} h_{2}^{2} I_{0}^{2}\left(\alpha_{n} h_{2}\right) \ddot{w}_{d}^{*}\left(\alpha_{n}, t\right)+m_{1} \int_{0}^{h_{2}} \ddot{w}_{s} y_{n} r \mathrm{~d} r=0
$$

Making use of (B.17) and (B.22), the last term on the left hand side of (B.24) is expressed by

$$
\int_{0}^{h_{2}} \ddot{w}_{s} y_{n} r \mathrm{~d} r=\frac{2}{h_{2}} \sum_{k_{n}} \frac{f^{*}\left(k_{n}\right)}{J_{1}\left(k_{n} h_{2}\right)} \frac{k_{n}^{3} I_{0}\left(\alpha_{n} h_{2}\right)}{\left(k_{n}^{4}-\alpha_{n}^{4}\right) \alpha_{n}^{2}} \ddot{\ddot{g}}\left(k_{n}, t\right)
$$

where $\bar{g}\left(k_{n}, t\right)$ are given in (21). Furthermore, from (B.23) we obtain a differential equation for $w_{d}^{*}$ as follows

$$
\ddot{w}_{d}^{*}+\frac{\alpha_{n}^{4}}{m_{1}} w_{d}^{*}+\sum_{k_{n}} \frac{\alpha_{n}^{2}}{\sqrt{m_{1}}} F\left(k_{n}, \alpha_{n}\right) \ddot{\bar{g}}\left(k_{n}, t\right)=0
$$

where

$$
F\left(k_{n}, \alpha_{n}\right)=\frac{2}{h_{2}^{3}} \frac{\sqrt{m_{1}} k_{n}^{3} f^{*}\left(k_{n}\right)}{J_{1}\left(k_{n} h_{2}\right) I_{0}\left(\alpha_{n} h_{2}\right)\left(k_{n}^{4}-\alpha_{n}^{4}\right) \alpha_{n}^{2}}
$$

Using eqn (21) and the following formula

$$
\frac{1}{24}+2 \sum_{m=1}^{\infty} \frac{\left[(-1)^{m}-1\right]}{(m \pi)^{4}}=0
$$

the initial conditions for function $\bar{g}$ are given by 


$$
\left.\bar{g}\right|_{t=0}=0,\left.\quad \dot{\bar{g}}\right|_{t=0}=0
$$

Consequently, using eqn (B.26) we obtain the transforming coefficient $w_{d}^{*}\left(\alpha_{n}, t\right)$ as follows

$$
w_{d}^{*}\left(\alpha_{n}, t\right)=C_{n}(t) \cos \left(\frac{\alpha_{n}^{2}}{\sqrt{m_{1}}} t\right)+D_{n}(t) \sin \left(\frac{\alpha_{n}^{2}}{\sqrt{m_{1}}} t\right)
$$

where

$$
\begin{aligned}
& C_{n}(t)=\int_{0}^{t} \sum_{k_{n}} F\left(k_{n}, \alpha_{n}\right) \ddot{\ddot{g}}\left(k_{n}, t^{\prime}\right) \sin \left(\frac{\alpha_{n}^{2}}{\sqrt{m_{1}}} t^{\prime}\right) \mathrm{d} t^{\prime} \\
& D_{n}(t)=-\int_{0}^{t} \sum_{k_{n}} F\left(k_{n}, \alpha_{n}\right) \ddot{\ddot{g}}\left(k_{n}, t^{\prime}\right) \cos \left(\frac{\alpha_{n}^{2}}{\sqrt{m_{1}}} t^{\prime}\right) \mathrm{d} t^{\prime}
\end{aligned}
$$

Let us introduce the notations $L_{1}(\eta)$ and $L_{2}(\eta)$

$$
\begin{aligned}
& L_{1}(\eta)=\int_{0}^{t} e^{-\eta t^{\prime}} \sin \left(\frac{\alpha_{n}^{2}}{\sqrt{m_{1}}} t^{\prime}\right) \mathrm{d} t^{\prime} \\
& L_{2}(\eta)=\int_{0}^{t} e^{-\eta t^{\prime}} \cos \left(\frac{\alpha_{n}^{2}}{\sqrt{m_{1}}} t^{\prime}\right) \mathrm{d} t^{\prime}
\end{aligned}
$$

which could be easily integrated as

$$
\begin{aligned}
& L_{1}(\eta)=\frac{1}{1+\frac{1}{\eta^{2}} \frac{\alpha_{n}^{4}}{m_{1}}}\left[\frac{1}{\eta^{2}} \frac{\alpha_{n}^{2}}{\sqrt{m_{1}}}-\frac{1}{\eta} e^{-\eta t} \sin \left(\frac{\alpha_{n}^{2}}{\sqrt{m_{1}}} t\right)-\frac{1}{\eta^{2}} \frac{\alpha_{n}^{2}}{\sqrt{m_{1}}} e^{-\eta t} \cos \left(\frac{\alpha_{n}^{2}}{\sqrt{m_{1}}} t\right)\right] \\
& L_{2}(\eta)=\frac{1}{1+\frac{1}{\eta^{2}} \frac{\alpha_{n}^{4}}{m_{1}}}\left[\frac{1}{\eta}-\frac{1}{\eta} e^{-\eta t} \cos \left(\frac{\alpha^{2}}{\sqrt{m_{1}}} t\right)+\frac{1}{\eta^{2}} \frac{\alpha_{n}^{2}}{\sqrt{m_{1}}} e^{-\eta t}\left(\frac{\alpha_{n}^{2}}{\sqrt{m_{1}}} t\right)\right]
\end{aligned}
$$

Finally, through (B.31)-(B.36) we obtain

$$
\begin{aligned}
w_{d}^{*}\left(\alpha_{n}, t\right)=\left[\sum_{k_{n}} F\left(k_{n}, \alpha_{n}\right) E_{1}\left(\alpha_{n}, k_{n}, t\right)\right] \cos \left(\frac{\alpha_{n}^{2}}{\sqrt{m_{1}}} t\right) & \\
- & {\left[\sum_{k_{n}} F\left(k_{n}, \alpha_{n}\right) E_{2}\left(\alpha_{n}, k_{n}, t\right)\right] \sin \left(\frac{\alpha_{n}^{2}}{\sqrt{m_{1}}} t\right) }
\end{aligned}
$$

and the dynamic deflection $w_{d}$ can be written as 


$$
\begin{aligned}
& w_{d}=\sum_{\alpha_{n}}\left\{\left[\sum_{k_{n}} F\left(k_{n}, \alpha_{n}\right) E_{1}\left(\alpha_{n}, k_{n}, t\right)\right] \cos \left(\frac{\alpha_{n}^{2}}{\sqrt{m_{1}}} t\right)-\left[\sum_{k_{n}} F\left(k_{n}, \alpha_{n}\right) E_{2}\left(\alpha_{n}, k_{n}, t\right)\right]\right. \\
& \left.\sin \left(\frac{\alpha_{n}^{2}}{\sqrt{m_{1}}} t\right)\right\}\left[I_{0}\left(\alpha_{n} r\right)-\frac{I_{0}\left(\alpha_{n} h_{2}\right)}{J_{0}\left(\alpha_{n} h_{2}\right)} J_{0}\left(\alpha_{n} r\right)\right]
\end{aligned}
$$

where

$$
\begin{aligned}
E_{1}\left(\alpha_{n}, k_{n}, t\right)=\frac{1}{24}\left[A^{2} L_{1}(A)\right. & \left.-B^{2} L_{1}(B)\right]+2 \sum_{m=1}^{\infty} \frac{\left[(-1)^{m}-1\right]}{(m \pi)^{4}}\left\{A^{2} L_{1}(A)-B^{2} L_{1}(B)\right. \\
& \left.-\left(\frac{m \pi}{h_{1}}\right)^{2}\left[\frac{A^{2} L_{1}(A)-C^{2} L_{1}(C)}{C-A}-\frac{\left.B^{2} L_{1}(B)-C^{2} L_{1}(C)\right]}{C-B}\right]\right\} \\
E_{2}\left(\alpha_{n}, k_{n}, t\right)=\frac{1}{24}\left[A^{2} L_{2}(A)\right. & \left.-B^{2} L_{2}(B)\right]+2 \sum_{m=1}^{\infty} \frac{\left[(-1)^{m}-1\right]}{(m \pi)^{4}}\left\{A^{2} L_{2}(A)-B^{2} L_{2}(B)\right. \\
& \left.-\left(\frac{m \pi}{h_{1}}\right)^{2}\left[\frac{A^{2} L_{2}(A)-C^{2} L_{2}(C)}{C-A}-\frac{B^{2} L_{2}(B)-C^{2} L_{2}(C)}{C-B}\right]\right\}
\end{aligned}
$$

The calculated results in terms of the solution (B.38) are shown in Figs 15-17.

\section{References}

Anderson, C.E., Bodner, S.R., 1988. Ballistic impact: the status of analytical and numerical modeling. Int. J. Impact Engng 7, 9-35.

Boley, B.A., Weiner, J.H., 1960. Theory of Thermal Stress. John Wiley and Sons, Inc.

Boustie, M., Cottet, F., 1991. Experimental and numerical study of laser induced spallation into aluminum and copper targets. J. Appl. Phys. 69, 7533-7538.

Chan, C.L., Mazumder, J., 1987. One-dimensional steady-state model for damage by vaporization and liquid expulsion due to laser-material interaction. J. Appl. Phys. 62, 4579-4586.

Das, S., 1983. Vibrations of polygonal plates due to thermal shock. J. Sound Vibrat. 89, 471-476.

Duan, Z.P., Zhou, Y.C., Xie, B.M., 1994. On laser-induced reverse plugging effect. In Zheng, Z.M. et al. (Eds.), Proceedings of IUTAM Symp. on Impact Dyn. Peking University Press, pp. 176-186.

Eliezer, S., Gilath, I., Bar-Noy, T., 1990. Laser induced spall in metals : experiment and simulation. J. Appl. Phys. 67 , $715-724$.

Fox, J.A., Barr, D.N., 1973. Laser-induced shock effects in plexiglass and 6061-T6 aluminium. Appl. Phys. Lett. 22, 594-596.

Garrison, J.N., 1976. Thermal stresses as laser heating damage mechanism, AD/A-034877.

Hartley, K.A., Duffy, J., Hawley, R.H., 1987. Measurement of the temperature profile during shear band formation in steels deforming at high strain rates. J. Mech. Phys. Solids 35, 283-301.

Jenq, S.T., Goldsmith, W., Kell, J.M., 1988. Effect of target bending in normal impact of a flat-ended cylindrical projectile near the ballistic limit. Int. J. Solids Struct. 24, 1243-1266.

Kar, A., Mazumder, J., 1990. Two-dimensional model for material damage due to melting and vaporization during laser irradiation. J. Appl. Phys. 68, 3884-3891.

Larson, A.R., 1974. Calculation of laser induced spall in aluminium targets. LA-5619. 
Liss, J., Goldsmith, W., Kell, J.M., 1983. A phenomenological penetration model of plates. Int. J. Solids Struct. 1, 321341.

Shoukry, M., Nair, S., Kalpakjian, S., 1991. Effect of shear deformation on the dynamic plastic bending of metallic plates during normal penetration. Int. J. Engng. Sci. 29, 1035-1052.

Smurov, I., Covelli, L., Tagirov, K., Aksenov, L., 1992. Peculiarities of pulse laser alloying: influence of spatial distribution of the beam. J. Appl. Phys. 71, 3147-3158.

Tauchert, T.R., 1989. Thermal shock of orthotropic rectangular plates. J. Thermal Stress 12, 241-258.

Zehnder, A.T., Rosakis, A.J., 1991. On the temperature distribution at the vicinity of dynamically propagating cracks in 4340 steel. J. Mech. Phys. Solids 39, 385-415.

Zhou, Y.C., Duan, Z.P., Xie, B.M., 1997a. Long pulsed laser induced reverse bulging and plugging. Int. J. Engng. Sci. $15,613-621$.

Zhou, Y.C., Duan, Z.P., Yang, Q.B., 1998. Shear deformation analysis on laser-induced reverse bulging and plugging. Int. J. Non-Linear Mechanics 33, 433-446.

Zweig, A.D., 1991. A thermo-mechanical model for laser ablation. J. Appl. Phys. 70, 1684-1691. 